\title{
Simultaneous processing of visual and spatial stimuli in pigeons
}

\author{
PHILIPP J. KRAEMER \\ Purdue University, West Lafayette, Indiana \\ DWIGHT S. MAZMANIAN \\ Concordia University, Montreal, Quebec, Canada \\ and \\ WILLIAM A. ROBERTS \\ University of Western Ontario, London, Ontario, Canada
}

\begin{abstract}
Pigeons were trained to symbolically match comparison stimuli to either visual sample stimuli presented on a center key or to spatial sample stimuli presented on side keys. Tests were carried out in which visual and spatial cues were simultaneously presented in compound and short-term memory was probed for either visual or spatial information. Symmetrical interference with the matching of visual and spatial components of compounds was found when the visual and spatial cues were presented on separate keys. However, when visual and spatial cues were superimposed on the same side key, no interference was observed relative to element control tests. Discussion of these findings focuses on accounts in terms of limited processing capacity, coding decrement, and receptor orientation mechanisms.
\end{abstract}

A number of recent experiments have used delayed matching-to-sample procedures to examine attentional processes in pigeons. The typical procedure has been to require pigeons initially to learn two identity matchingto-sample problems. For example, pigeons would be trained to peck the red or green comparison stimulus on one of two side keys that matched the color just previously seen on a center key. On a concurrently learned lineorientation problem, each trial began with the presentation of a vertical or horizontal white line on an otherwise darkened center key, followed by a choice between matching and nonmatching lines on side keys. A pigeon's ability to process two signals simultaneously then was examined by briefly presenting a compound on the center key consisting of either a red or green background and either a vertical or horizontal white line superimposed on the background. Short-term memory for the colored background was probed by presenting red and green comparison keys; memory for line orientation was tested by presenting vertical and horizontal white lines on comparison keys with identical dark backgrounds. Experiments of this nature revealed a deficit in pigeons' ability to match both the color and line components of the compound relative to control trials in which only the element stimulus was presented as a sample (Lamb \& Riley, 1981; Maki \& Leith, 1973; Maki, Riley, \& Leith, 1976; Roberts \&

Support for this research was provided by Grant A7894 from the Natural Sciences and Engineering Research Council of Canada. Requests for reprints should be sent to William A. Roberts, Department of Psychology, University of Western Ontario, London, Ontario, Canada N6A $5 \mathrm{C} 2$.
Grant, 1978). It was argued that divided or shared attention had been demonstrated in pigeons (Riley, 1984; Riley \& Leith, 1976; Riley \& Roitblat, 1978).

Unfortunately, several problems have arisen with the attentional interpretation of these experiments. For one, it has been pointed out that the deficit found with compound samples could arise from generalization decrement within trials (Cox \& D'Amato, 1982; Roberts \& Grant, 1978). Generalization decrement could arise from the change in appearance of a line or color when presented in compound as a sample and then presented alone as a comparison stimulus. No such loss in accuracy should arise on control trials, since the sample and correct comparison stimuli are identical. A second difficulty is the observation that the deficit found on compound-sample trials relative to element-sample trials does not interact with length of exposure to the sample stimulus. If the compound-element difference arises from a limited channel that cannot effectively process two messages simultaneously, the effect should be most marked when samples are shown briefly and tend to disappear when samples are given long exposures. With extended presentation, the memory system should be able to process both signals adequately. Yet, the deficit found with compound stimuli has been found to persist over sample-exposure durations as long as $30 \mathrm{sec}$ (Lamb \& Riley, 1981; Roberts \& Grant, 1978; Santi, Grossi, \& Gibson, 1982).

Very recently, Grant and MacDonald (1986) provided still another challenge to the notion of divided attention by arguing that the difference found between compound and element tests arose from a generalization decrement 
between the training and testing phases of experiments. Grant and MacDonald found that pigeons trained to match compound sample stimuli and then tested with compounds and elements showed better matching with compounds than with elements. They suggested that pigeons do not decompose line-color compounds into components and that the divided-attention effect arises from generalization decrement between the element-sample stimuli used in training and the compound-sample configurations used in testing.

As an alternative to the identity matching-to-sample procedure for studying attention in pigeons, a symbolic matching procedure was used recently in two sets of studies by Kraemer and Roberts $(1985,1987)$. Symbolic matching has certain advantages over identity matching for studying animals' ability to process simultaneously presented sample stimuli. One is that symbolic matching eliminates any possible interpretation of findings in terms of within-trials generalization decrement. Since sample and comparison stimuli come from different dimensions and have no similarity relationship, any loss of accuracy on compound tests cannot be explained by differential similarity of sample to test stimuli. A second advantage is that symbolic matching allows the investigator to examine attentional effects for a much broader range of environmental stimuli than those provided by colors and patterns on a sample key.

In the Kraemer and Roberts (1985) study, pigeons' ability to process simultaneously presented visual and auditory signals was studied. One group of birds learned a problem in which pecking a comparison key that contained a horizontal line was rewarded after presentation of a red ambient light and pecking a key that contained a vertical line was rewarded after a green ambient light; in another problem, the same birds were trained to peck a yellow comparison key after hearing a $300-\mathrm{Hz}$ tone and a blue comparison key after hearing a $3000-\mathrm{Hz}$ tone. On compound test trials, tone and color samples were presented simultaneously, and memory for either tone or color was probed by presenting the appropriate comparison stimuli. The results showed clearly that the tone had no influence on pigeons' ability to match colors; on the other hand, the presence of light strongly reduced matching to the tone. In the similar Kraemer and Roberts (1987) study, compounds of bright and dim houselight brightness and horizontal and vertical line patterns were used. As in the auditory-visual processing experiments, it was found that one dimension (brightness) strongly interfered with the other dimension (line orientation) but was little affected by the presence of the second dimension.

Kraemer and Roberts's $(1985,1987)$ findings differ from the divided-attention effects obtained with identity matching. Studies using identity matching to line-color compounds typically have shown symmetrical interference effects, with equal deficits in the matching of line and color components. The Kraemer and Roberts studies revealed asymmetrical deficits, with one dimension totally dominating the other. Unlike the identity-matching experiments, these findings are not easily accounted for by generalization decrement. Within-trials generalization decrement is ruled out by the fact that stimuli from different dimensions were used as samples and comparison stimuli. Generalization decrement between training and test trials appears unlikely, because this account predicts interference with both components of the test compound, not just one. Kraemer and Roberts (1987) argued that their findings demonstrated restricted processing, or a hardwired tendency of animals to process only one stimulus dimension when presented with certain stimulus compounds.

In the experiment reported here, symbolic matching was used to examine simultaneous processing of spatial and visual information. Pigeons were trained in a three-key chamber to symbolically match comparison stimuli presented on side keys to spatial stimuli that consisted of the illumination of either the right or left side key with white light. As a second problem, the same pigeons were trained to match different comparison stimuli to visual cues presented on the center key. When both problems were well learned, simultaneous processing of visual and spatial information was studied by presenting a visual-spatial compound and then presenting appropriate comparison stimuli to test for a match to either the spatial or the visual component of the compound. Comparison of performance on compound trials with that on element control trials allowed us to examine possible divided-attention effects. In addition, tests were carried out with two types of spatial-visual compounds. The compounds consisted of either separated cues presented on separate keys or superimposed cues presented on the same key. This comparison allowed us to examine the possibility that compound processing might depend upon the way in which sample stimuli were combined.

Conflict between spatial and visual cues has often been a factor in theories of discrimination learning. Spence (1936) held that imbalances between spatial response strengths influenced visual discrimination learning, and Sutherland and Mackintosh (1971) argued that the base strength of the spatial analyzer was stronger than that of the visual analyzer. In comparative studies of animal intelligence, Bitterman (1965) has suggested that advanced behavioral processes might appear earlier in phylogeny on spatial discrimination problems than on visual discrimination problems. Because these various theoretical considerations suggested that spatial cues might have priority over visual cues, we anticipated that visual cues might suffer more interference in a compound presentation than would spatial cues.

\section{METHOD}

\section{Subjects and Apparatus}

The subjects were 5 adult White King pigeons that had received no training prior to being trained to match spatial and visual cues. They were maintained at $80 \%$ of their free-feeding weights throughout the experiment. The birds were housed with other pigeons in a room where the temperature was kept at $22^{\circ} \mathrm{C}$ and where they 
had constant access to water and health grit. Windows in the housing room provided daylight illumination, and overhead fluorescent lights were turned on at 8:30 a.m. and turned off at 10:30 p.m. Testing was carried out between 9:00 a.m. and 1:00 p.m. for 6 days each week.

All testing was conducted in a standard operant chamber for pigeons that measured $31 \times 35.5 \mathrm{~cm}$ (floor dimensions) $\times 35.3 \mathrm{~cm}$ (wall height). The front wall of the chamber contained a row of three pecking keys, level with a pigeon's head and spaced $8 \mathrm{~cm}$ apart, center to center. An electromechanical food hopper provided mixed grain through a $6 \times 6 \mathrm{~cm}$ opening centered on the front wall below the pecking keys. Multistimulus projectors, located behind each key, presented solid blue, yellow, red, green, or white fields on each key; in addition, patterns consisting of either a white triangle or three white dots in a diagonal row could be projected against a black background. All trial event programming and response recording was controlled by a Commodore 64 computer and a locally constructed computer interface.

\section{Procedure}

All 5 subjects initially were trained to match comparison stimuli to spatial sample stimuli. Each daily session consisted of 48 trials of 0-sec delayed matching. For 3 subjects, the spatial sample stimuli were mapped onto the triangle and diagonal-dot patterns as comparison stimuli; for the other 2 subjects, spatial samples were mapped onto red and green fields. Each trial began with $4 \mathrm{sec}$ of whitelight illumination of the right or left key. Immediately after termination of the white sample light, the two side keys were simultaneously illuminated with the appropriate comparison stimuli; a peck to the triangle or the red field yielded $2 \mathrm{sec}$ of grain reinforcement after illumination of the left side key, and a peck to the diagonal dots or the green field yielded reinforcement after illumination of the right side key. Immediately after reinforcement, or after response to an incorrect side key, a 20 -sec intertrial interval spent in darkness began. Two quasi-random sequences of 48 trials were used on alternate days; these sequences allowed the same sample stimulus and the same position of the correct comparison stimulus to occur only three times in a row. Within each sequence, the spatial sample appeared on the left on 24 trials and on the right on 24 trials. Within the 24 trials devoted to each spatial position of the sample, one left-right arrangement of the comparison stimuli occurred on 12 trials and the reverse arrangement occurred on the remaining 12 trials. Each pigeon was trained on the spatial matching problem until it reached a criterion of $90 \%$ correct choices or better in two successive sessions.

When a subject had achieved criterion performance on the spatial sample problem, it began training on the visual sample problem. The visual samples were blue and yellow fields presented on the center key. For the 3 birds trained to match the triangle and diagonaldot patterns to spatial samples, red and green fields were the comparison stimuli, with red the reinforced choice after a blue sample and green the reinforced choice after a yellow sample. In the case of the 2 pigeons trained to match red and green comparison stimuli to spatial samples, the triangle and diagonal-dot patterns were the comparison stimuli, with the triangle correct after a blue sample and the dots correct after a yellow sample. Each of the four different combinations of sample stimulus and left-right arrangement of the comparison stimuli occurred on 12 trials within the daily session of $\mathbf{4 8}$ trials. There were two quasi-random sequences of trials used on alternate days, with the orders of trials subject to the same constraints used in the sequences of spatial sample trials. Training on the visual problem was carried to the same $90 \%$ criterion as had the spatial problem. During visual training, the subjects occasionally were given a session of spatial training to ensure that a high level of performance was maintained on the original problem. The subjects were around $90 \%$ accurate on these spatial retraining sessions.
Experimental testing began immediately after an animal had reached criterion on the visual sample problem. Three experiments were carried out to examine the processing of spatial-visual compounds. The first two experiments independently examined performance with separated and superimposed compounds, and the third experiment directly compared these two conditions. Only the third experiment is reported here, since it captures the important findings of all the experiments.

On alternate days, the subjects were tested on separated and superimposed compounds. Test sessions contained 480 -sec delayed matching trials, with 24 trials devoted to element control tests and 24 trials devoted to compound tests. The element control trials were identical to training trials, with spatial samples tested on 12 trials and visual samples tested on the other 12 trials. On separated compound trials, the sample consisted of $4 \mathrm{sec}$ of illumination of the left or right side key with white light at the same time as the center key was illuminated with a visual stimulus. On superimposed compound trials, either the right or the left side key contained the visual cue; a single side key thus presented visual information by its content and spatial information by its position. Immediately after termination of either type of spatial-visual compound, appropriate comparison stimuli were presented on side keys to probe memory for either spatial or visual information. Spatial information was probed on 12 trials within a session, and visual information was probed on the other 12 compound trials.

Each of four different quasi-random sequences of 48 trials was used once within a block of 4 days. Two of these sequences contained separated compound trials, and the other two contained superimposed compound trials. For each type of compound, there were 16 different possible combinations of spatial and visual sample cues with left-right arrangements of spatial and visual comparison stimuli, and each of these combinations was tested three times over two sets of 48 trials. Birds were tested for 20 sessions, with sequences containing separated compounds used on odd-numbered sessions and sequences containing superimposed compounds used on evennumbered sessions.

\section{RESULTS}

The percentage of correct choices on element and compound trials is shown in Figure 1. There was no significant difference between percent correct choices on element trials run in sessions with superimposed compounds

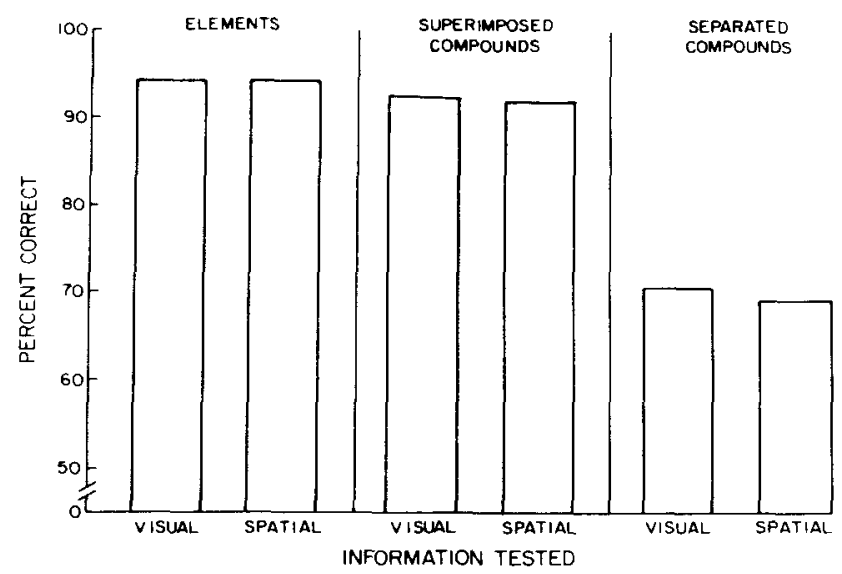

Figure 1. Percentage of correct choices made on trials with element-sample stimuli and on trials with compound samples consisting of either superimposed or separated spatial and visual stimuli. 
and those run in sessions with separated compounds. Thus, all of the element trials were pooled to provide the spatial and visual element bars in the left panel of Figure 1 . On trials with superimposed compounds, pigeons were about $92 \%$ accurate and only slightly below the $94 \%$ accuracy seen on element control trials. By contrast, accuracy on trials with separated compounds was essentially $70 \%$, far below that with superimposed compounds. In the case of elements and both compounds, there was virtually no difference in accuracy on tests of spatial and visual information.

An analysis of variance was performed that contained three factors, type of sample stimulus (elements, superimposed compounds, and separated compounds), type of information tested (visual vs. spatial), and subjects. The analysis revealed a significant effect of type of sample stimulus $[F(2,8)=132.96, p<.01]$ and nonsignificant effects of information tested $(F<1.00)$ and the interaction of type of sample stimulus $x$ information tested $(F<1.00)$. A Newman-Keuls test with alpha set at .05 was used to compare the mean percentage correct across the three types of sample stimuli. It was found that the element and superimposed-compound conditions were both significantly higher than the separated compound condition, but that they did not differ significantly from one another.

\section{DISCUSSION}

These findings clearly indicate that the ease with which pigeons can process spatial-visual compounds is heavily dependent upon how the compound is presented. Considerable interference with the matching of compound elements was found when the elements were presented at separated positions in space. When the visual color cue was placed on a side key, the pigeons were able to match either the position in space of the side key or the color on the key with accuracy that was very near that obtained on element trials. The important implication of this finding is that visual and spatial information can be processed simultaneously by the pigeon with no loss of either kind of information. Furthermore, the symmetrical nature of the interference effects seen with separated compounds suggests that the pigeons did not preferentially process spatial or visual information. As mentioned earlier, this experiment captures the results of two previous experiments carried out with these pigeons. The first experiment used separated spatial-visual compounds, and the second used superimposed compounds. In both experiments, the effects reported here appeared immediately on the initial sessions of testing.

Evidence appears to be accumulating that weighs against a limited-capacity, or bottleneck, model of attention (Riley $\&$ Leith, 1976). The divided-attention effect found with line-color compound-sample stimuli does not interact with sample duration time as the model predicts (Lamb \& Riley, 1981; Roberts \& Grant, 1978; Santi et al., 1982). Recent findings suggest that asymmetrical selective atten- tion effects may be found with visual-auditory compounds (Kraemer \& Roberts, 1985) and brightness-pattern compounds (Kraemer \& Roberts, 1987). The current finding that no interference was found with superimposed spatialvisual cues appears to offer further difficulties for the limited-capacity model.

If channel capacity is limited, how can spatial and visual information share the same channel without interference, while color and line information cannot? One solution would be to suggest that spatial and visual information have separate channels, whereas color and line information do not. Such a suggestion would significantly complicate the attentional model and sacrifice parsimony. Without some independent evidence as to which sources of information have independent channels and which share a channel, the theory cannot predict when interference will or will not appear.

As another possible account of these data within a limited-channel-capacity framework, it might be argued that the superimposed spatial-visual compounds were processed as integral stimuli (Garner, 1970; Riley \& Leith, 1976). Separable stimuli are easily analyzed, whereas integral stimuli are difficult to analyze and are said to form a new unitary dimension. Lamb and Riley (1981) presented pigeons with element stimuli on a key that consisted of either a red or orange field or three horizontal or vertical white lines on a black ground. In compound testing, color and line stimuli were combined in different ways. The lines and colors were presented either spatially separated from one another by a short or long distance on the center key or were presented within the same square area. When presented within the same area, the lines were either superimposed upon the colored field or were unified with the color. That is, both white lines and a colored background appeared in the superimposed condition, whereas only colored horizontal or vertical bars appeared in the unified condition. Although all compound conditions yielded significantly worse performance than did element tests, the unified condition showed less interference than the superimposed and separated conditions. Lamb and Riley suggested that the unified condition involved integral stimuli and therefore could pass more easily through a limited-channel information processing system than could the separate dimensions extracted from the superimposed- and separated-cue conditions.

This account of Lamb and Riley's (1981) data might be extended to the finding presented here by arguing that our superimposed and separated conditions represent integral and separable stimuli, with the integral stimulus passing more readily through the information bottleneck. However, we feel that there is a fundamental problem with this account of the present data as well as with Lamb and Riley's data. For animals to effectively choose the correct comparison stimulus on test trials, they must extract line orientation or color information from a compound or its representation at some stage in processing. If animals code prospectively (Grant, 1981; Honig \& Thompson, 
1982), then the sample compound must be analyzed into color and line-orientation components in order to retrieve the correct response codes. If retrospective memory is used (Urcuioli \& Zentall, 1986), the bird must use the comparison stimuli to retrieve the correct color or line orientation from the memory of the sample stimulus. If integral stimuli are difficult to analyze, the use of an integral sample stimulus should increase interference on compound test trials, not reduce it. The pigeon's task may be seen as similar to that of a human trying to sort compound integral stimuli into separate dimensions. Interference arises when the subject has to abstract the brightness or saturation of integral dimensions but not of separated dimensions (Garner, 1970). It may be argued then that pigeons faced with superimposed spatial-visual compounds would suffer interference because such compounds require extra time and cognitive effort to analyze into their separate dimensions. It is therefore not clear that identifying superimposed spatial-visual compounds as integral stimuli will allow these data to be understood within a limited-capacity model.

A quite different approach was taken by Grant and MacDonald (1986), who assumed that pigeons do not break down compounds into their elements but process them only as a single configuration. Through training with elements, pigeons learn to retrieve appropriate response codes when presented with a sample stimulus. When elements are placed in compounds, the efficiency of the coding process is disturbed to the extent that the compounds used in testing look dissimilar to the elements used in training.

From the point of view of Grant and MacDonald's coding decrement model, the failure to observe interference with superimposed spatial-visual cues is also puzzling. Presentation of the colored visual sample on a side key should appear to be a significant change from presentation of the color on the center key or a white light on the side key. An appeal to the integral versus separable nature of stimulus compounds might be made here on the basis of an argument that differs from that discussed above with respect to limited-capacity theory. It might be argued that integral compounds appear more similar to element sample stimuli than do separable compounds. For example, in the present experiment, moving a blue key light from a center key to a side key may have little changed the pigeon's perception of the color of the key and its ability to form the appropriate response code, and changing a white light on the left key to a blue light may have little effect on a pigeon's perception of the position of the key. In support of such an assumption, Garner (1970) suggests that changes along the dimensions of integral stimuli are perceived as producing smaller psychological distances than are changes along the dimensions of separable stimuli. In general, spatial models suggest that integral stimuli vary according to a Euclidean model, whereas separable stimuli vary according to a city-block model.

We prefer to interpret the results presented here and other recent findings largely in terms of peripheral orient- ing mechanisms. Brown, Cook, Lamb, and Riley (1984) recently have provided evidence to suggest that a large part of the decrement in accuracy seen with separated stimulus elements may arise from selective peripheral processing. That is, pigeons tend to peck selectively at one element of a compound. Although we did not measure pecking rates on sample keys in the experiment reported, keypecking has been measured in other experiments in our laboratory using separated spatial-visual compounds. These observations show clearly that pigeons tend to spend the sample presentation time pecking on either the spatial or the visual component of the compound. If the pigeon then is tested on the component it pecked, accuracy is very high, essentially at the level achieved on element tests. If the comparison stimuli probe memory for the nonpecked component, however, accuracy is no higher than chance. We suggest that the lower level of performance seen in our separated-compound condition was largely the result of selective orientation to one component of the compound. In further support for this interpretation, we can multiply the probability that the pecked component will be tested by the level of accuracy achieved on element trials $(.50 \times .94=.47)$ and add this to the probability that the nontested component will be pecked multiplied by the chance level of accuracy $(.50 \times .50=.25)$. The sum of these products is .72 , a value close to the .70 level of performance found with separated compounds.

Thus, when presented with two stimuli at different spatial locations, pigeons orient selectively and may peck at one stimulus and not the other. This form of differential orientation accounts for the interference seen in the separated-compound condition. Although no strong preferences for orientation toward the center or side key appeared in this experiment, in other situations using symbolic matching, innate preferences for orienting toward one stimulus over another may come into play. Kraemer and Roberts (1987) found that pigeons' ability to match line-orientation sample stimuli declined substantially when these stimuli were placed in compound with a houselight sample stimulus. Correlated with this drop in accuracy was a significant decrease in frequency of pecks to the center key containing the line stimulus. Processing may have been largely restricted to the houselight because pigeons spent most of the sample-presentation period looking at the houselight. In the case of simultaneous presentation of visual and auditory stimuli (Kraemer \& Roberts, 1985), it is unlikely that differential peripheral orientation was at work, but these findings may be understood by an innate sensory gating mechanism that tends to shut down auditory processing when salient visual information is presented (Posner, Nissen, \& Klein, 1976).

\section{REFERENCES}

Bitterman, M. E. (1965). The evolution of intelligence. Scientific American, 212, 92-100.

Brown, M. F., Cook, R. G., Lamb, M. R., \& Riley, D. A. (1984)

The relation between response and attentional shifts in pigeon com- 
pound matching-to-sample performance. Animal Learning \& Behavior, 12, 41-49.

Cox, J. K., \& D'Amato, M. R. (1982). Matching to compound samples by monkeys (Cebus apella): Shared attention or generalization decrement. Journal of Experimental Psychology: Animal Behavior Processes, 8, 209-225.

GarNer, W. R. (1970). The stimulus in information processing. American Psychologist, 25, 350-358.

GRANT, D. S. (1981). Short-term memory in the pigeon. In N. E. Spear \& R. R. Miller (Eds.), Information processing in animals: Memory mechanisms (pp. 227-256). Hillsdale, NJ: Erlbaum.

Grant, D. S., \& MacDonald, S. E. (1986). Matching to element and compound samples in pigeons: The role of sample coding. Journal of Experimental Psychology: Animal Behavior Processes, 12, 160-171.

Honig, W. K., \& Thompson, R. K. R. (1982). Retrospective and prospective processing in animal working memory. In G. H. Bower (Ed.), The psychology of learning and motivation: Advances in research and theory (Vol. 16, pp. 239-283). New York: Academic Press.

Kraemer, P. J., \& Roberts, W. A. (1985). Short-term memory for simultaneously presented visual and auditory signals in the pigeon. Journal of Experimental Psychology: Animal Behavior Processes, 11, 137-151.

Kraemer, P. J., \& Roberts, W. A. (1987). Restricted processing of simultaneously presented brightness and pattern stimuli in pigeons. Animal Learning \& Behavior, 15, 15-24.

LAMB, M. R., \& RiLEY, D. A. (1981). Effects of element arrangement on the processing of compound stimuli in pigeons (Columba livia). Journal of Experimental Psychology: Animal Behavior Processes, 7, 45-58.

MAKI, W. S., \& LEITH, C. R. (1973). Shared attention in pigeons. Journal of the Experimental Analysis of Behavior, 19, 345-349.
MAKI, W. A., Riley, D. A., \& LeITH, C. R. (1976). The role of test stimuli in matching-to-compound samples. Animal Learning \& Behavior, 4, 13-21.

Posner, M. I., Nissen, M. J., \& Klein, R. M. (1976). Visual dominance: An information-processing account of its origins and significance. Psychological Review, 83, 157-171.

RILEY, D. S. (1984). Do pigeons decompose stimulus compounds? In H. L. Roitblat, T. G. Bever, \& H. S. Terrace (Eds.), Animal cognition (pp. 333-348). Hillsdale, NJ: Erlbaum.

RILEY, D. A., \& LEITH, C. R. (1976). Multidimensional psychophysics and selective attention in animals. Psychological Bulletin, 83, 138-160.

Riley, D. A., \& RoITBLAT, H. L. (1978). Selective attention and related cognitive processes in pigeons. In S. H. Hulse, H. Fowler, \& W. K. Honig (Eds.), Cognitive processes in animal behavior (pp. 249-276). Hillsdale, NJ: Erlbaum.

RoBERTS, W. A., \& GRANT, D. S. (1978). Interaction of sample and comparison stimuli in delayed matching to sample with pigeons. Journal of Experimental Psychology: Animal Behavior Processes, 4, 68-82.

Santi, A., Grossi, V., \& Gibson, M. (1982). Differences in matching to-sample performance with element and compound sample stimuli in pigeons. Learning \& Motivation, 13, 240-256.

SPENCE, K. W. (1936). The nature of discrimination learning in animals. Psychological Review, 43, 427-449.

Sutherland, N. S., \& MACKINTOSH, N. J. (1971). Mechanisms of animal discrimination learning. New York: Academic Press.

Urculol, P. J., \& Zentall, T. R. (1986). Retrospective coding in pigeons' delayed matching-to-sample. Journal of Experimental Psychology: Animal Behavior Processes, 12, 69-77.

(Manuscript received January 29, 1987; revision accepted for publication June 25, 1987.) 